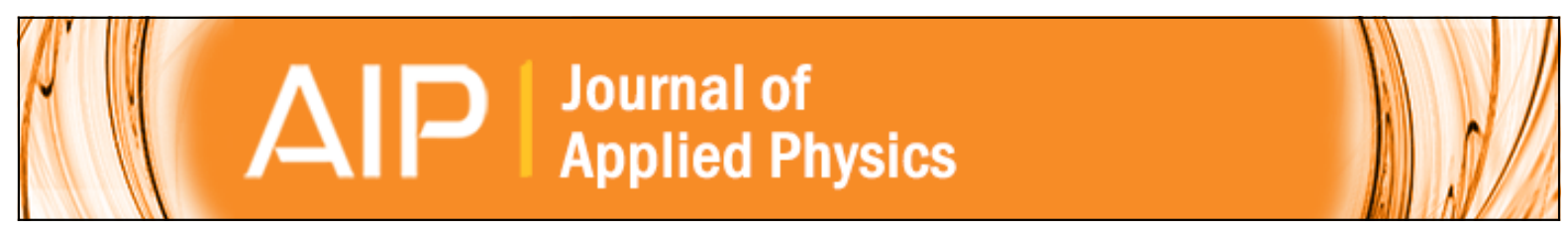

\title{
Surface Barriers on Zinc Oxide
}

R. C. Neville and C. A. Mead

Citation: Journal of Applied Physics 41, 3795 (1970); doi: 10.1063/1.1659509

View online: http://dx.doi.org/10.1063/1.1659509

View Table of Contents: http://scitation.aip.org/content/aip/journal/jap/41/9?ver=pdfcov

Published by the AIP Publishing

\section{Articles you may be interested in}

Magnetic tunnel junctions with a zinc oxide-cobalt oxide composite tunnel barrier

Appl. Phys. Lett. 86, 112505 (2005); 10.1063/1.1882762

Study of oxidized cadmium zinc telluride surfaces

J. Vac. Sci. Technol. A 15, 850 (1997); 10.1116/1.580719

The sublimation of basal surfaces of zinc oxide

J. Chem. Phys. 63, 2646 (1975); 10.1063/1.431658

dc triode sputtered zinc oxide surface elastic wave transducers

J. Appl. Phys. 44, 1061 (1973); 10.1063/1.1662307

Oxide Stabilized Surface Barrier Detectors

Rev. Sci. Instrum. 32, 1253 (1961); 10.1063/1.1717216

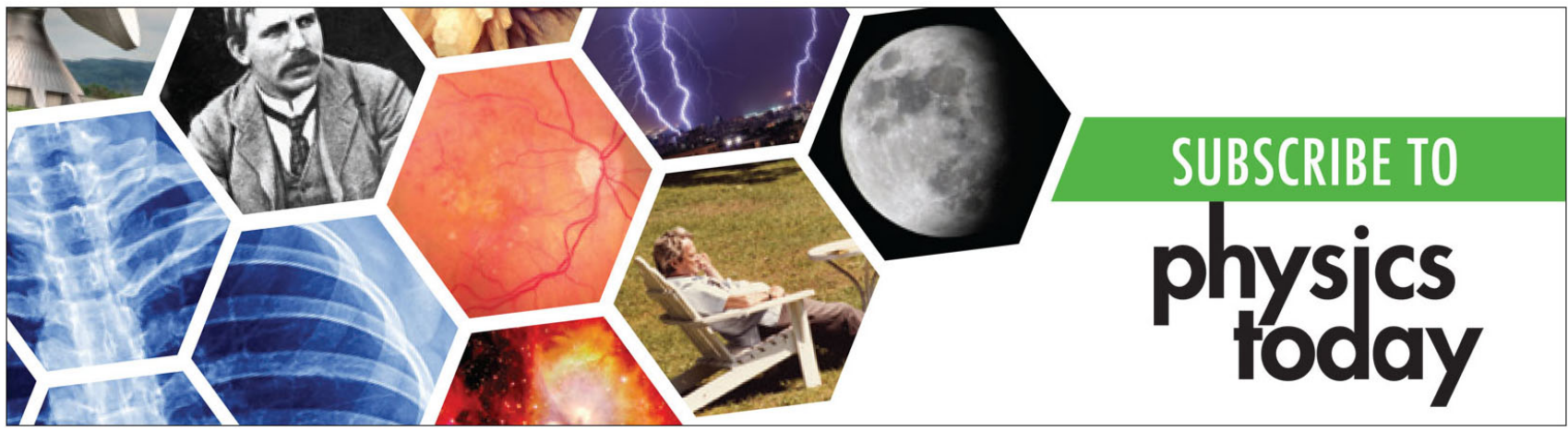




\title{
Surface Barriers on Zinc Oxide
}

\author{
R. C. Neville AND C. A. Mead \\ Department of Electrical Engineering, California Institute of Technology, Pasadena, California 91109
}

(Received 9 February 1970; in final form 16 April 1970)

\begin{abstract}
The surface barrier systems consisting of gold and palladium on chemically prepared zinc oxide have been investigated in detail. Surface barrier energies have been determined by photoresponse, forward current versus voltage, thermal activation energy, and capacitance-voltage methods. Agreement in barrier energies obtained by the four methods is excellent. The barrier energy for gold is $0.66 \mathrm{eV}$ and for palladium is $0.60 \mathrm{eV}$. Forward current-voltage characteristics were in quantitative agreement with simple Bethe diode theory as modified by the presence of image force lowering. The reverse current-voltage characteristic is in quantitative agreement with that expected from the simple image force lowering of the barrier, over a bias range of from 0.1 to $3 \mathrm{~V}$. Carrier concentration derived from resistivity and Hall measurements agreed with that obtained from capacitance-voltage measurements. We believe this represents the first comprehensive study where such quantitative consistency has been demonstrated on a compound semiconductor barrier system. Existence of a deep level trap is indicated via the effects on capacitance measurements.
\end{abstract}

\section{INTRODUCTION}

Zinc oxide as a material has been known since the Bronze Age. ${ }^{1}$ It was not until relatively recently that detailed investigations into the electronic properties of this hexagonal crystal have been conducted. Rupprecht ${ }^{2}$ and Bogner ${ }^{3}$ determined the mobility of bulk samples of zinc oxide. The dielectric constant for zinc oxide was determined by Collins and Kleinman ${ }^{4}$ and an effective mass for the charge carrier was measured by Dietz et al..$^{5} \mathrm{Mead}^{6}$ determined the surface barrier energies of several metals on vacuum-cleaved zinc oxide. The barrier energy for gold was determined to be $0.71 \mathrm{eV}$ and $0.65 \mathrm{eV}$ was obtained as barrier energy for palladium.

We report here the results of an extensive investigation of the barrier-semiconductor systems formed by gold and palladium on zinc oxide. Barrier energies were determined by photoresponse, forward current-voltage, thermal activation energy, and capacitance-voltage methods. Forward and reverse current-voltage characteristics were studied and are analyzed in terms of the simple Bethe diode theory, ${ }^{7}$ as modified by the presence of image force lowering. ${ }^{8,9}$

\section{SAMPLE PREPARATION}

Undoped hexagonal zinc oxide crystals with free electron concentrations between $1 \times 10^{16}$ and $2 \times 10^{17}$ per cubic centimeter were used. ${ }^{10}$ The crystals were first cleaned by immersion in concentrated phosphoric acid for a period of $15 \mathrm{~min}$, followed by a 10 -min soak in concentrated hydrochloric acid and a rinse in flowing deionized water. The crystals were dried in a jet of dry air. The samples were then placed in a vacuum chamber at a pressure of $10^{-6}$ Torr or less. Using a heated tungsten filament approximately $1000 \AA$ of gold or palladium was evaporated on the sample through a fine mesh. The active barrier area was nominally $100 \mu$ in diameter. Contact to the evaporated metal barriers was made by a fine gold wire probe. Ohmic contact to the bulk crystals was made using $10 \%$ silver and $90 \%$ indium solder.
On three of the four crystal samples the same crystal was given both gold and palladium barriers.

\section{RESULTS AND INTERPRETATION}

\section{Bulk Measurements}

Mobility and resistivity measurements were made on the bulk zinc oxide crystals. Mobility was determined by a Hall measurement using a $4.7 \mathrm{kG}$ permanent magnet. Resistivity measurements were made using four soldered contacts at both room $\left(296^{\circ} \mathrm{K}\right)$ and at liquid-nitrogen $\left(77^{\circ} \mathrm{K}\right)$ temperatures. The resistivity was then combined with the mobility data to derive an effective donor concentration of

$$
N_{d}=1 / \rho \mu q,
$$

where $N_{d}$ is the donor concentration, $\rho$ is the measured resistivity, $\mu$ is the Hall mobility, assumed equal to the conductivity mobility, and $q$ is the electronic charge.

For a typical crystal the measured mobility was 200 $\mathrm{cm}^{2} / \mathrm{V} \mathrm{sec}$, in good agreement with values quoted in the literature (Refs. 2,3). The resistivity of this sample at room temperature was $1.13 \Omega$-cm. At liquid-nitrogen temperatures the measured resistivity was $1.98 \Omega-\mathrm{cm}$. From the resistivity and mobility at room temperature the net donor concentration was calculated to be $2.9 \times$ $10^{16} / \mathrm{cc}$.

\section{Surface Barriers}

Surface barrier energies were determined using four independent techniques. The methods used were: photoresponse, zero-voltage forward current intercept, thermal activation energy, and capacitance variation with applied voltage.

\section{Photoresponse}

The barrier energy was determined by a measurement of the short circuit photocurrent using light entering the crystal from the barrier contact side (a front wall configuration). A tungsten-halide lamp was used in conjunction with a Gaertner Quartz Prism Monochro- 


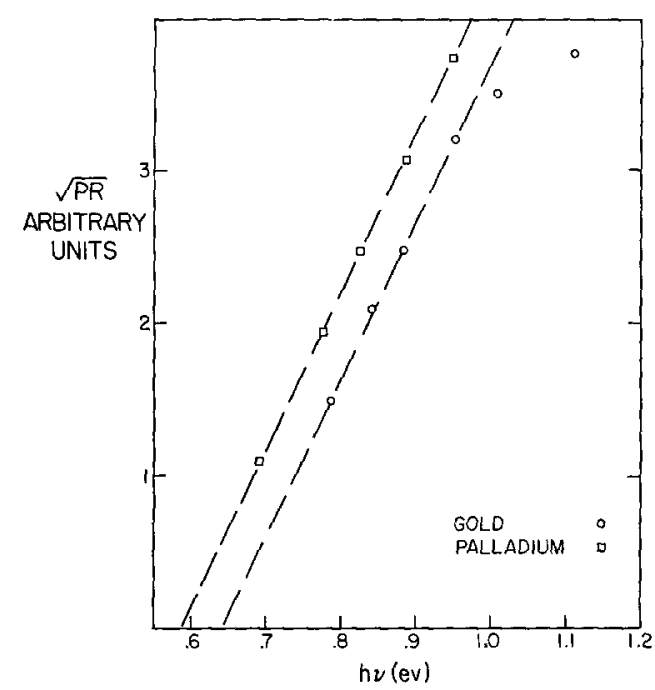

Fig. 1. Square root of photo current per incident photon as a function of photon energy for gold and palladium surface barriers on zinc oxide.

nometer. From simple Fowler theory, the barrier energy is the intercept for zero response of the plot of the square root of the response (photo current per incident photon) versus the photon energy.

In Fig. 1 we present the photoresponse data from typidal sample crystals. Four crystals were used with measurements being taken on between 5 and 15 barriers per crystal. The surface barrier energies, were found to be $0.645 \pm 0.04 \mathrm{eV}$ for gold and $0.59 \pm 0.04 \mathrm{eV}$ for palladium.

\section{Forward Current versus Voltage}

Typical curves of forward current density versus forward voltage are presented in Fig. 2 for gold and palladium barriers on chemically prepared zinc oxide. The area of each device is given in the figure. Current measurements were made with a three-lead configuration to eliminate errors due to potential drop at the bulk crystal contact.

The current-voltage characteristic, for thermiomic current and voltages in excess of a few $k T / q$ is given by

$$
J=J_{0} \exp (q V / n k T),
$$

where $J$ is the current density, $V$ is the applied voltage, $k$ is the Boltzmann constant, $T$ is the absolute temperature, and

$$
J_{0}=A^{*} T^{2} \exp (-q \phi / k T),
$$

where $\phi$ is the surface barrier energy, $A^{*}$ is the Richardson constant corresponding to the effective mass of the material, and

$$
A^{*}=m^{*} q k^{2} / 2 \pi^{2} \hbar^{3}
$$

where $m^{*}$ is the effective mass taken as $0.38 m_{e}$ after Ref. $5, m_{e}$ is the rest mass of the electron, and $\hbar$ is Planck's constant divided by $2 \pi$.
The factor $(n)$ in Eq. (2) is treated in Henish ${ }^{8}$ and in Sze. ${ }^{11}$ It arises here from the change in $\phi$ with applied voltage.

$$
n=1+(\partial \phi / \partial V),
$$

where after Ref. 9,

$$
\begin{aligned}
\Delta n(\text { image force })= & \partial \phi / \partial V \\
= & \frac{1}{4}\left(q^{3} N d / \epsilon_{0}^{3} 8 \pi^{2} \epsilon_{o p}{ }^{2} \epsilon_{D C}\right)^{1 / 4} \\
& \quad \times[\phi-V-\zeta-(k T / q)]^{-3 / 4},
\end{aligned}
$$

where $\Delta n$ is the deviation of $n$ from unity, $\epsilon_{0}$ is the permittivity of free space, and $\epsilon_{0 \mathrm{p}}$ is the relative permittivity of zinc oxide at optical frequencies. After Ref. 12 this is set at 4. $\epsilon_{D C}$ is the low-frequency relative permittivity of zinc oxide. After Collins and Kleinman (Ref. 4) this is taken at $8 . V$ is the applied voltage and $\zeta$ is the Fermi energy below the conduction band edge. $\zeta$ is given by

$$
\zeta=k T / q \ln N_{C} / N_{D}
$$

where $N_{C}$ is the conduction band effective density of states and where

$$
N_{C}=\left(\pi^{1 / 2} / 2 / \pi^{2} \hbar^{3}\right)\left(m_{e}^{*}\right)^{3 / 2}(k T)^{3 / 2} .
$$

Combining Eqs. (3) and (4) one can write for the diode nonideality factor

$$
n=1+\Delta n \text { (image force). }
$$

The value of $n$ for forward voltages between 0.05 and $0.15 \mathrm{~V}$ is found to be $1.05 \pm 0.05$. This value is in agreement with that expected as a result of image force lowering from the Eqs. (4) and (6) using the carrier concentration determined from resistivity and Hall effect measurements.

Using Eq. (2a) the surface barrier energies were calculated from current-voltage data taken on some

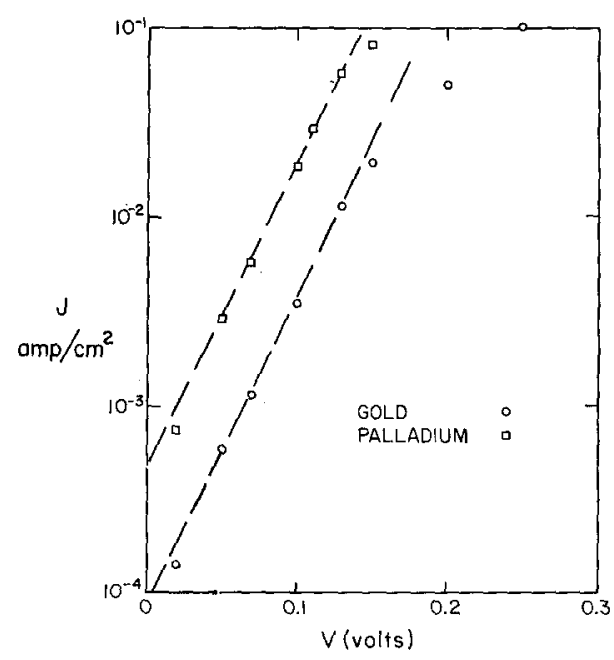

Frg. 2. Typical forward current density as a function of applied voltage for gold and palladium surface barriers on zinc oxide at $300^{\circ} \mathrm{K}$. 
40 individual barriers on 4 crystals. The energies obtained in this manner are $0.66 \pm 0.03 \mathrm{eV}$ for gold and $0.60 \pm 0.04 \mathrm{eV}$ for palladium on zinc oxide.

\section{Thermal Activation Energy}

A third measurement of the surface barrier energy was obtained from the measurement of reverse current as a function of the temperature at fixed bias voltage. A Peltier cooler was employed and the results of the measurement are presented in Fig. 3. The activation energy was determined from the relationship:

$$
\phi_{A}=(-k / q)\left[\partial \ln I_{s}(V) / \partial 1 / T\right]-(2 k T / q),
$$

where $I_{8}(V)$ is the current at some fixed bias $V$.

The thermal activation energies obtained on chemically treated zinc oxide for 40 individual barriers on four crystals are $0.70 \pm 0.04 \mathrm{eV}$ for gold and $0.63 \pm 0.04$ $\mathrm{eV}$ for palladium.

The thermal activation energy determined from Eq. (7) is expected to be higher than the barrier energy due to the change in barrier energy with temperature. In general, the barrier energy is not constant with temperature, but over a small temperature range is expected to vary approximately as follows:

$$
\phi(T)=\phi_{0}+A_{1} T,
$$

where $\phi_{0}$ is the barrier energy at the measurement temperature and $A_{1}$ is the temperature coefficient of the barrier energy in the vicinity of the measurement temperature.

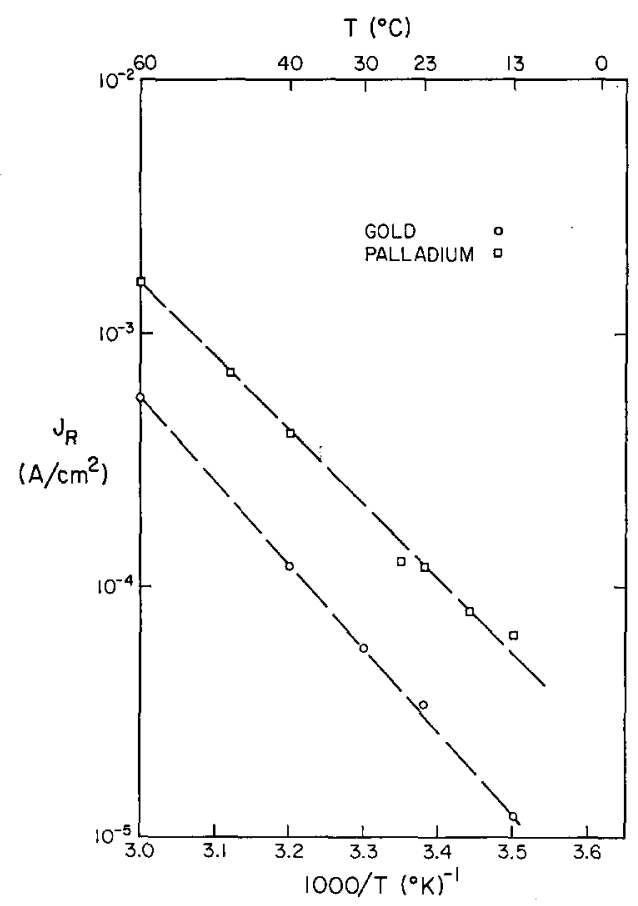

FIg. 3. Current density as a function of temperature for a reverse bais of $1 \mathrm{~V}$.

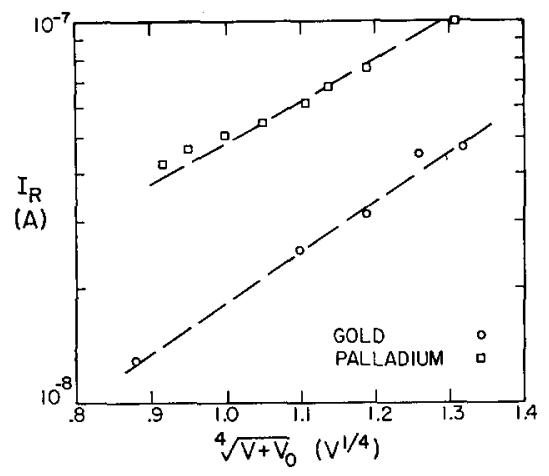

Fig. 4. Reverse current as a function of applied voltage for gold and palladium surface barriers on zinc oxide.

In covalent semiconductors the presence of surface states fixed the Fermi level, at the surface, relative to one band edge. In such instances it has been found that the barrier energy varies in temperature as does the bandgap. ${ }^{13}$ An ionic semiconductor has virtually no surface states. The Fermi level, at the surface, is free to move between conduction and valence band edges, depending upon the work function of the metal employed. Zinc oxide is an ionic semiconductor with no surface states. ${ }^{6,14}$ The variation of surface barrier energy with temperature is then expected to be proportional to that fraction of the forbidden gap occupied by the barrier.

$$
A_{1}=\partial \phi / \partial T=\left(\phi / E_{g}\right)\left(\partial E_{g} / \partial T\right)<0,
$$

where $E_{g}$ is the forbidden gap.

The bandgap energy for zinc oxide is $3.435 \mathrm{eV}$ at $1.2^{\circ} \mathrm{K} .{ }^{15}$ The barrier energy for gold and palladium on zinc oxide has been determined by photoresponse and forward current versus voltage techniques. The change in the forbidden gap with temperature has been measured by Watanabe and Wada ${ }^{16}$ to be $-8 \times 10^{-4} \mathrm{eV} /{ }^{\circ} \mathrm{K}$ in the vicinity of room temperature. From Eq. (9) $A_{1}$ is $-1.57 \times 10^{-4} \mathrm{eV}$ for gold and $-1.43 \times 10^{-4} \mathrm{eV}$ for palladium. Use of these values in Eq. (8) together with the barrier values attained from Eq. (7) results in substantial agreement for values of $\phi$ as determined by photoresponse, forward current, and thermal activation energy (see Table I).

\section{Reverse Current}

The voltage dependence of the reverse current characteristic, at fixed temperature, as a function of the fourth root of the applied voltage is displayed in Fig. 4. At a fixed temperature the reverse current as a function of the applied voltage is expected to be nonconstant. The ideal, constant reverse current, independent of applied bias, of the Bethe diode theory is modified by the presence of the image force field lowering which causes the barrier energy to change with applied voltage. ${ }^{9}$ The theoretical slope of the logarithm of the reverse current 
TABLE I. Measured barrier energies on zinc oxide.

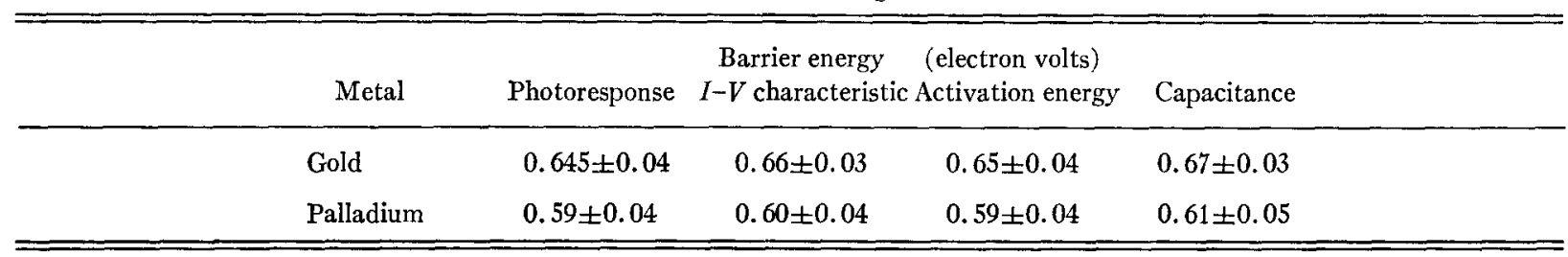

versus voltage is given by

$\left[\partial \ln I s(V) / \partial\left(V_{0}-V\right)^{1 / 4}\right]$

where

$$
=(q / k T)\left(q^{3} N_{d} / 8 \pi^{2} \epsilon_{0}^{3} \epsilon_{\mathrm{op}}^{2} \epsilon_{D C}\right)^{1 / 4},
$$

$$
V_{0} \text { is } \phi-\zeta-k T / q \text {. }
$$

The slope as given by Eq. (10) is also plotted in Fig. 4 . The agreement between theory and experiment for both gold and palladium surface barriers is seen to be good over a range in bias between 0.1 and $3 \mathrm{~V}$.

\section{Capacitance Studies}

The fourth measurement of the surface barrier energies was made using the variation of barrier capacitance with applied voltage at a frequency of $1 \mathrm{MHz}$. In Fig. 5 we present $1 / C^{2}$ versus applied voltage for two typical surface barriers under the conditions of darkness and illumination with $0.5-\mu$ light.

This technique not only yields the surface barrier energy but allows determination of the effective donor concentration of that part of the crystal directly under the individual barrier. Following Goodman ${ }^{17}$ the effective donor concentration is

$$
N_{d}=\left(-2 / q \epsilon_{D C} \epsilon_{0}\right)\left[\partial V / \partial(S / C)^{2}\right],
$$

where $S$ is the barrier area and $C$ is the measured capacitance at the applied voltage.

The extrapolated intercept $V_{0}$ of a plot of $1 / C^{2}$ versus the applied voltage is related to the surface barrier energy by the equation

$$
V_{0}=\phi-\zeta-k T / q
$$

In the derivation of Eqs. (11) and (12) the absence of trapping was assumed. The effect of empty traps is to increase the measured donor concentration. Thus the measurement of capacitance under illumination of sufficiently short wavelength is expected to yield values of donor concentration in excess of the true value. The measured intercept will be lower than the true value of barrier energy by the Fermi level and a thermal term.

Table II presents, in summary form, the donor concentrations and extrapolated intercepts $V_{0}$ for the several zinc oxide crystals studied. An average of seven barriers of each type were measured and both the averages and scatter for each sample is given.

The sample marked palladium- $A$ was a crystal possessing a much higher impurity concentration. level than did the other crystals. The surface barrier energies as determined by all four techniques on this heavily doped sample were in agreement with the barrier energies obtained from the other, less heavily doped, samples. No systematic relationship between surface barrier energy and impurity concentration was found.

The values of carrier concentration obtained from the capacitance-voltage data on individual surface barriers under conditions of no illumination are in good agreement with the values obtained by Hall and resistivity measurements. For example, sample number 3 was found earlier to have an effective donor concentration of $2.9 \times 10^{16} / \mathrm{cc}$. The capacitance-voltage technique yields a value of $(3 \pm 0.3) \times 10^{16} / \mathrm{cc}$.

The Fermi level was calculated to be $0.135 \mathrm{eV}$ below the conduction band using Eqs. (5a) and (5b) with a capacitance derived carrier concentration of $3 \times 10^{16} / \mathrm{cc}$. From this and Eq. (12) the barrier energies were calculated to be $0.67 \pm 0.03 \mathrm{eV}$ for gold on zinc oxide and $0.61 \pm 0.05 \mathrm{eV}$ for palladium.

\section{Capacitance and Traps}

The capacitance of the surface barriers measured was a function of the wavelength and intensity of the illumination. The samples were illuminated by a strong fluorescent source with a number of filters of a differing cutoff wavelength. An increase in the capacitance was experienced for wavelengths in the $1-1.5-\mu$ range. From this data the trap level energy depth was estimated to be between 0.8 and $1.2 \mathrm{eV}$.

The deep trap density can be estimated by comparing the donor density under illumination and no illumination. A lower bound for the trap density $N_{t}$ can be estimated by using

$$
\left.N_{t} \approx N_{d} \text { (illuminated }\right)-N_{d} \text { (dark) } .
$$

The lower bound trap densities are estimated from Table II to be $2 \times 10^{15} / \mathrm{cc}$ for all samples except palladium- $A$. For palladium- $A$ the trap density is estimated to be at least $2 \times 10^{16} / \mathrm{cc}$. Based on limited data it appears that the trap density fluctuates with the carrier concentration and has a magnitude approximately onetenth the donor concentration. Further investigation of the trapping site will be required to determine the exact nature of the trap, its erergy level, cross section, and origin.

\section{Barrier Energy Summary}

In Table I the values of surface barrier energy resulting from the four measurement techniques are presented. 
The photoresponse data and $I-V$ characteristic data are the averages and scatter obtained from the 40 barriers tested. The activation energy is corrected for variation in barrier energy with temperature and the capacitance data is adjusted for individual barrier donor concentration as noted. We find the barrier energy of gold on chemically prepared zinc oxide to be $0.66 \pm 0.04$ $\mathrm{eV}$ and the surface barrier energy of palladium on chemically prepared zinc oxide to be $0.60 \pm 0.04 \mathrm{eV}$.

Agreement in values of $\phi$ as determined by all four techniques is excellent and consistent. The individual scatter in data from crystal to crystal was of the same order as the scatter from crystal to crystal.

\section{CONCLUSIONS}

The metal-semiconductor systems composed of goldzinc oxide and palladium-zinc oxide have been investigated.

The surface barrier energies of gold and palladium on chemically prepared zinc oxide have been measured by the current-versus-voltage, photoresponse, thermal activation energy, and capacitance-versus-voltage techniques. The barrier energies obtained are in close agreement for the four methods.

The surface-barrier current-voltage characteristics have been found to be in quantitative agreement with simple Bethe diode theory as modified by image force lowering. Agreement is good in the forward direction for biases between 0.05 and $0.15 \mathrm{~V}$. Under reverse bias good agreement was obtained between 0.1 and $3.0 \mathrm{~V}$. The forward portion of the characteristic displays a

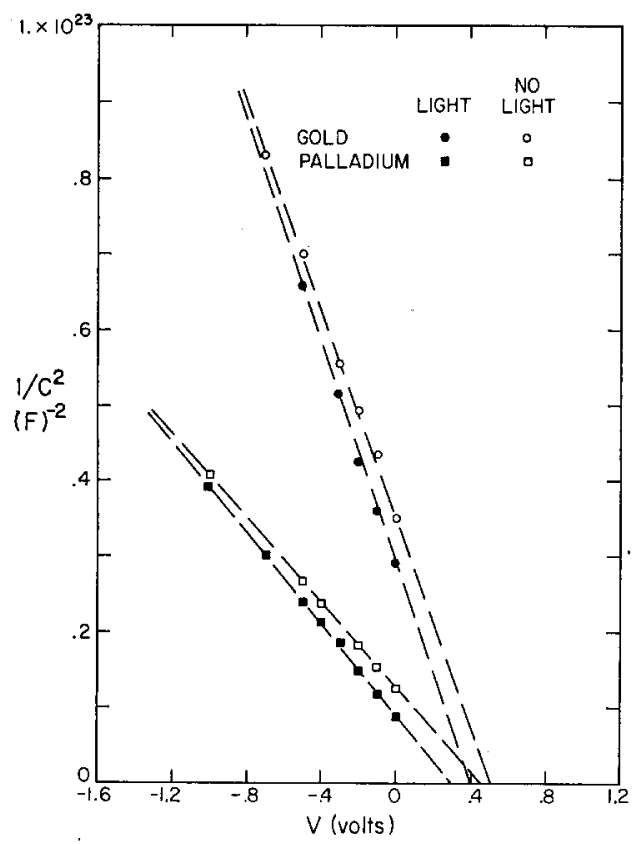

FIG. 5. Barrier capacitance as a function of applied bias voltage. Bias polarity is that of the metal relative to the $\mathrm{ZnO}$. Measurement frequency was $1 \mathrm{MHz}$. "Light" values were obtained under intense illumination. (See text.)
TABLE II. Donor concentration and infinite capacitance intercept for zinc oxide samples.

\begin{tabular}{llll}
\hline \hline Metal & & $\begin{array}{c}\text { Donor } \\
\text { concentration } \\
N^{*}\left(\mathrm{~cm}^{-3}\right)\end{array}$ & $\begin{array}{c}\text { Intercept } \\
V_{0}(\mathrm{~V})\end{array}$ \\
\hline Gold & Yllumination & $(3.3 \pm 2) \times 10^{16}$ & $0.42 \pm 0.03$ \\
Gold & No & $(3.1 \pm 2) \times 10^{16}$ & $0.51 \pm 0.03$ \\
Palladium & Yes & $(3.2 \pm 3) \times 10^{16}$ & $0.35 \pm 0.03$ \\
Palladium & No & $(3.0 \pm 3) \times 10^{16}$ & $0.45 \pm 0.03$ \\
Palladium- $A$ & Yes & $(2.0 \pm 0.2) \times 10^{17}$ & 0.39 \\
Palladium- $A$ & No & $(1.8 \pm 0.3) \times 10^{17}$ & 0.45 \\
\hline
\end{tabular}

diode nonideality factor $n$ of $\mathbf{1 . 0 5}$. It is shown that this value is in agreement with image force lowering of the barrier. The reverse portion of the current-voltage curve is shown to be thermal in nature and in quantitative agreement with image force lowering voltage dependence of the surface barrier.

The crystals investigated were found to have mobilities of $200 \mathrm{~cm}^{2} / \mathrm{V} \mathrm{sec}$ and carrier concentrations between $1 \times 10^{16}$ and $2 \times 10^{17} / \mathrm{cc}$. The mobility values were in agreement with those reported in the literature..$^{3,4}$ The initial bulk determination of the carrier concentration by resistivity and Hall measurements was confirmed for individual barriers on a local basis by the capacitance variation with applied voltage.

Throughout the discussion an effective mass of $0.38 m_{e}$ was used in accord with Dietz et al..$^{6}$ This gave good agreement for forward current versus voltage derived surface barrier energies and when computing the Fermi level.

The existence of traps in the forbidden gap was demonstrated. Trap density is estimated to be $2 \times 10^{15}$ $2 \times 10^{16} / \mathrm{cc}$. There appears to be a relatively constant ratio of 0.1 between trap density and effective donor density. The trap level is estimated to lie at a depth of approximately $1 \mathrm{eV}$ below the conduction band edge.

In conclusion, the agreement between theory and experimental observation for the zinc oxide-gold, and zinc oxide-palladium surface barrier systems is striking. We believe this represents the first comprehensive study where such quantitative consistency has been demonstrated on a compound semiconductor barrier system.

\section{ACKNOWLEDGMENT}

During this work many fruitful discussions were held with Dr. T. McGill.

${ }^{1}$ H. E. Brown, Zinc Oxide Rediscovered (New Jersey Zinc Co., New York, 1957).

2 H. Rupprecht, J. Phys. Chem. Solids 6, 144 (1958).

${ }^{3}$ G. Hogner, J. Phys. Chem. Solids 19, 235 (1961).

4 R. J. Collins and D. A. Kleinman, J. Phys. Chem. Solids 11, 190 (1959).

${ }^{5}$ R. C. Dietz, J. J. Hopfield, and D. G. Thomas, J. Appl. Phys. 32, 2282 (1961). 
${ }^{6}$ C. A. Mead, Phys. Lett. 18, 218 (1965).

${ }^{7}$ H. A. Bethe, MIT Rad. Lab. Rept.: 43/12 (1942).

${ }^{8}$ H. K. Henisch, Rectifying Semiconductor Contacts (Clarendon, Oxford, 1957)

${ }^{9}$ S. M. Sze, C. R. Crowell, and D. Kahng, J. Appl. Phys. 35, 2534 (1964)

${ }^{10} \mathrm{R}$. D. Carlton (private communication).

${ }^{11}$ S. M. Sze, Physics of Semiconductor Devices (Wiley-Interscience, New York, 1969).

\author{
12 American Institute of Physics-Handbook (McGraw-Hill, \\ New York, 1957). \\ ${ }^{13}$ Crowell, S. M. Sze, and Spitzer, Appl. Phys. Lett. 4, 91 \\ (1964). \\ ${ }_{14}$ J. F. Dewald, Bell Syst. Tech. J. 39, 615 (1960). \\ ${ }_{15}$ Y. S. Park, C. W. Litton, T. C. Collins, and D. C. Reynolds, \\ Phys. Rev. 143, 512 (1966) \\ ${ }_{16}$ H. Watanabe and M. Wada, Jap. J. Appl. Phys. 3, 617 \\ (1964). \\ ${ }_{17}$ A. M. Goodman, J. Appl. Phys. 34, 329 (1963).
}

J OUR N AL OF A P PLIED. PHYSICS

VOL UME 41, N UMBER 9

A U G UST 1970

\title{
Accommodation of Misfit Across the Interface Between Crystals of Semiconducting Elements or Compounds
}

\author{
J. W. Matthews, S. Mader, and T. B. Light \\ IBM Watson Research Center, Yorktown Heights, New York 10598
}

(Received 6 March 1970)

\begin{abstract}
Misfit dislocations are often transported to the interface between crystals by glide. In materials with the diamond or sphalerite structures, glide is inhibited by the Peierls stress. The aim of this paper is to present a theory for misfit accommodation which includes the effect of the Peierls stress. It is used to explain the elastic strains present in films of germanium on gallium asrenide and to explain the reduction of these strains by a high-temperature anneal. The strains in germanium on gallium arsenide, and the effect of temperature upon them, are not explained by earlier theories for the accommodation of misfit between one crystal and another.
\end{abstract}

\section{INTRODUCTION}

In 1949 Frank and van der Merwe ${ }^{1}$ considered the accommodation of misfit across the interface between one crystal grown epitaxially on another. This work has since been extended by van der Merwe, ${ }^{2}$ Cabrera, ${ }^{3}$ and Jesser and Kuhlmann-Wilsdorf. ${ }^{4}$ They find it energetically favorable for the overgrowth to strain elastically so as to either reduce or eliminate the misfit between the two crystals. If misfit is reduced but not eliminated by elastic strain the remainder is accommodated by misfit dislocations. The division of misfit between dislocations and elastic strain depends upon the difference between the two bulk lattice parameters, on the thickness of the overgrowth, on the geometry of the dislocations, as well as on the bonding across the interface, and on the elastic constants of the two materials. Experimental confirmation of some of the predictions has been obtained from thin deposits of one fcc metal on another. ${ }^{5-9}$ It has also been found that fcc metal films thick enough for one to expect most of the misfit to be accommodated by dislocations have elastic strains significantly larger than the predicted values. ${ }^{6,10}$ This discrepancy between theory and experiment seems to result from work hardening during the relaxation of misfit strain.11

Studies of misfit accommodation in crystals with the diamond or sphalerite structures have not provided clear evidence in support of theoretical predictions. However, they have shown that misfit is either accommodated entirely by strain or is shared between dis- locations and strain..$^{12-14}$ Recently, Sugita, Tamara, and Sugawara ${ }^{14}$ have made two quantitative measurements which are in satisfactory agreement with van der Merwe's ${ }^{2}$ predictions. The aim of this paper is to show that agreement with the predictions of van der $\mathrm{Merwe}^{2}$ and others ${ }^{3,4}$ is expected under some conditions but not under others. The origin of the disagreement between theory and experiment is the large Peierls stress ${ }^{15}$ in crystals with the diamond or sphalerite structures. If the crystals are initially dislocation free, or almost so, then an additional deviation from theory is expected to be present.

\section{MISFIT DISLOCATIONS IN ADAMANTINE [CRYSTALS}

To calculate the way in which misfit is divided between dislocations and elastic strain we need to know the geometry of misfit dislocations and how they are formed. Observations made by optical microscopy, ${ }^{12}$ transmission electron microscopy, ${ }^{16}$ and $\mathrm{x}$-ray topography ${ }^{14,17}$ indicate that misfit dislocations in semiconductors glide from a free surface into the interface or junction region. ${ }^{16} \mathrm{~A}$ mechanism ${ }^{6,9}$ for this is shown in Fig. 1. Figure 1(a) shows a grown-in dislocation line which extends from crystal B through the interface and into crystal A. Figure 1(b) shows this dislocation after it has bowed under the influence of the misfit strain. In Fig. 1(c) a length of misfit dislocation line has been made by migration of the bowed dislocation. 